\section{EU-Arbeitsschutzrichtlinie über physikalische Auswirkungen Ausnahmeregelung für MRT gefordert}

Im Jahr 2004 hat die EU im Rahmen einer Gesamtstrategie zum Schutz von Arbeitnehmern vor physikalischen Auswirkungen neue Grenzwerte für elektromagnetische Felder beschlossen. Grundlage hierfür ist die so genannte EMF-Richtlinie. Die Einhaltung der Grenzwerte in Krankenhäusern und radiologischen Praxen würde aus Sicht von Ärzten den Einsatz von Magnetresonanztomografen (MRT) allerdings stark einschränken. Die Kritik der Ärzteschaft führte schließlich dazu, dass das Inkrafttreten der Richtlinie um 4 Jahre auf Ende April 2012 verschoben wurde. Nun soll es nach dem Willen der EU-Kommission und von EU-Abgeordneten eine Ausnahmeregelung für den medizinischen Bereich geben. Einige EU-Staaten, darunter Deutschland, sind hingegen anderer Ansicht.
„Europa ist führend in der MRT-Technologie und darf seinen Patienten den Zugang zu diesem herausragenden Bildgebungsverfahren nicht verwehren“, erklärt Professor Gabriel Krestin, führender Vertreter der Allianz für Kernspintomografie und Vizepräsident der Europäischen Gesellschaft für Radiologie. Die Allianz ist eine Koalition von EU-Parlamentariern, Patientengruppen, europäischen Wissenschaftlern und Ärzten, die gemeinsam versuchen, die von der EU-Richtlinie über physikalische Einwirkungen (kurz EMFRichtlinie) ausgehende Bedrohung der Anwendung von MRT für klinische und Forschungszwecke abzuwenden.

Als der für den Arbeitsschutz zuständige EU-Kommissar Vladimir Spidla im Jahr 2004 seinen ersten Entwurf für die Richtlinie 2004/40/EG präsentierte, formierte sich in den ärztlichen Fachkreisen alsbald Widerstand gegen die neuen Vorschrif- ten. Denn die Regelungen enthielten Grenzwerte, die eine Magnetresonanztomografie aus Sicht der Deutschen Röntgengesellschaft (DRG) praktisch unmöglich machen würden.

\section{Nutzung von MRT durch Grenzwerte gefährdet?}

„Die strengen Grenzwerte gefährden die Nutzung von MRT vor allem bei intraoperativen Verfahren und bei Untersuchungen von Kindern, älteren und sedierten Patienten“, sagt der Essener Radiologe und Präsident der DRG, Professor Dr. med. Michael Forsting. Denn Ärzte und andere medizinische Fachkräfte wären nach den neuen Regeln beim Einsatz am MRT Strahlenbelastungen ausgesetzt, die oberhalb der festgelegten Grenzwerte liegen. Die ordnungsgemäße Reinigung und Instandhaltung der MR-Geräte werde ebenfalls erschwert, informiert die Allianz.

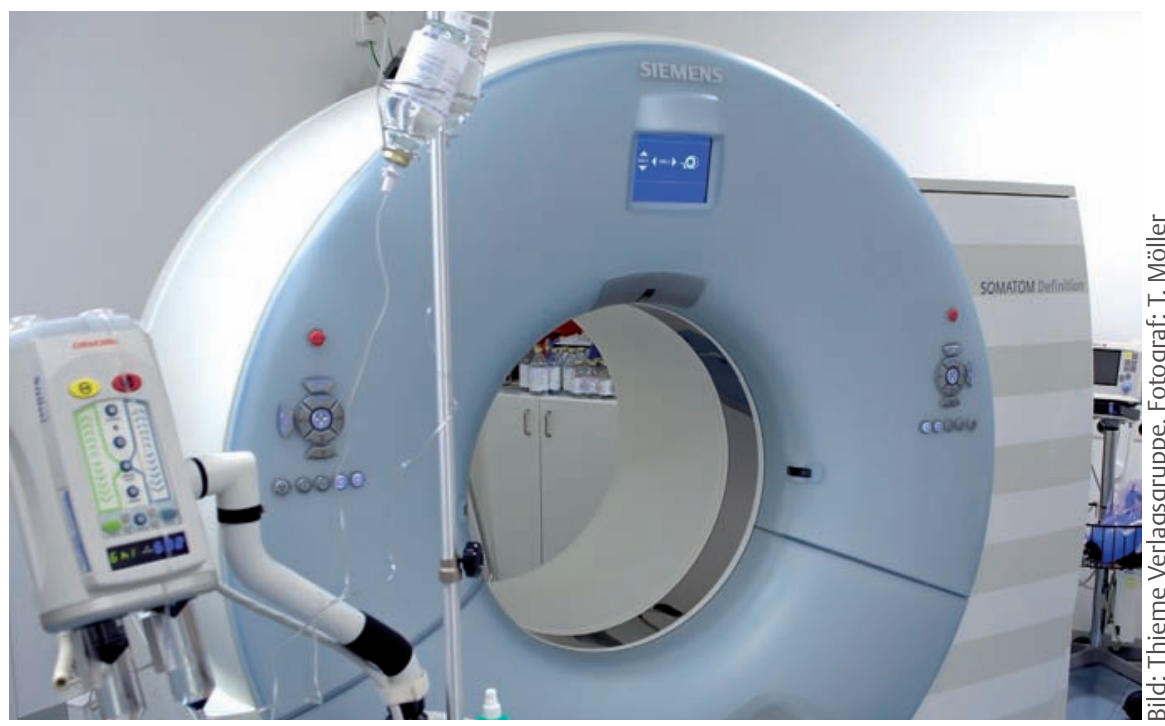

Europa ist derzeit führend auf dem Gebiet der Forschung und der Entwicklung dieser Technologie. Pro Jahr profitieren in der EU rund 8 Millionen Patienten von der Kernspintomografie. In den letzten 25 Jahren wurden weltweit nach Auskunft der DRG über 500 Millionen MRTUntersuchungen durchgeführt - ohne nachgewiesene negative Auswirkungen aufgrund von Belastungen durch elektromagnetische Felder. Die Gefahren seien allenfalls theoretischer Natur. Die Strahlenbelastung durch die Computertomografie oder das Röntgen, die als Alternative zum MRT infrage kämen, seien unverhältnismäßig höher, betont Forsting.

Die Proteste der Radiologen und der Allianz für Kernspintomografie zeigten schließlich Wirkung. EU-Kommissar Spidla zog seinen ursprünglichen Richtlinienvorschlag im Oktober 2007 zurück und versprach, die Vorschriften zu überarbeiten. Die Umsetzungsfrist wurde auf Ende April 2012 verschoben.

\section{Scheitert die Ausnahmeregelung}

für Medizin an der Blockadehaltung

einiger Länder?

Der neue Gesetzentwurf liegt seit Sommer letzten Jahres vor. Er sieht nun ganz im Interesse der Ärzte eine Ausnahmeregelung für die medizinische Nutzung von MRT vor. „Die Medizin kann mit dem überarbeiteten Vorschlag gut leben“, so Forsting. Auch beim Europaparlament (EP) stößt die überarbeitete Fassung auf Zustimmung. Ende Januar stimmte der Gesundheitsausschuss des EP der Ausnahmeregelung nahezu einstimmig zu. „Wir haben heute zwar die Ausnahme für 
die medizinischen Anwendungen beschlossen, aber dennoch sichergestellt, dass das medizinische Personal keiner noch so theoretischen Gefahr ausgesetzt ist," betont der CDU-Europaabgeordnete Dr. Horst Schnellhardt.

Ärzte und Politiker fürchten nun aber, dass die neuen Regelungen an der Blockadehaltung einiger EU-Mitgliedsländer im Ministerrat - darunter Deutschland scheitern könnten. Denn eine Handvoll EU-Regierungen lehnt es ab, für einen bestimmten Sektor ᄃ Ausnahmen von den Arbeitneh을 merschutzvorschriften zu gewähren, da dies dem Grundsatz der 흠 Gleichbehandlung widerspräche. „Mich überzeugen die Bedenken der Mitgliedstaaten nicht", so der CDU-Europaabgeordnete und Arzt, Dr. Peter Liese. „Es wäre verrückt, wenn die Mitgliedstaaten einen Vorschlag zum Bürokratieabbau, den die Kommission vorgelegt hat und den das Parlament unterstützt, auf Dauer verhindern würden.“

Aufgrund der unterschiedlichen Positionen im EP und im Ministerrat ist allerdings fraglich, ob die Richtlinie wie vorgesehen Ende April in Kraft treten kann. Denn das Parlament, dessen Plenum im März über die Neuregelungen abstimmt, muss sich mit den EURegierungen auf eine gemeinsame Fassung einigen. Das Bundesarbeitsministerium, das für Deutschland an den Verhandlungen im Rat teilnimmt, erklärte auf Anfrage, dass es schwierig sein wird, zügig eine abschließende differenzierte Bewertung aller offenen Fragen vornehmen zu können.

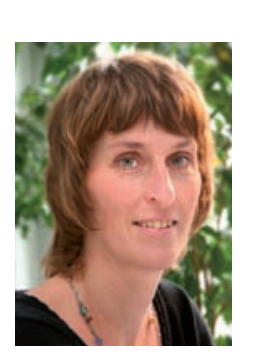

Korrespondenz

Petra Spielberg

Fachjournalistin

für Gesundheitsund Sozialpolitik

Wiesbaden/

Brüssel

Fax.: 0611/

98818512

p.spielberg@

t-online.de

Der Beitrag erschien zuerst in der Zeitschrift klinikarzt 2/2012. 